\title{
Effect of feeding high fat diet on reproductive parameters in male mice
}

\author{
Rosa J Samuel*1, Omana Joy ${ }^{2}$, P. Krishna Murthy ${ }^{3}$, Shruthi Iyer, S.G. Ramachandra and K. Thamaraiselvi ${ }^{1}$ \\ Central Animal Facility, Division of Biological Sciences, \\ Indian Institute of Science, Bangalore-560 012, Karnataka, India. \\ Received: 10-11-2017 \\ Accepted: 20-03-2018 \\ DOI: 10.18805/ijar.B-3528
}

\begin{abstract}
The present study attempts to chronicle the series of changes starting from the consumption of high fat diet till reproductive activity in male mice. A marginal increase in body weight was observed in male mice fed with high fat diet with 3-fold increase in total body fat and 1.4-fold increase in adipose tissue. A significant reduction in number and diameter of seminiferous tubules were observed in high fat diet fed mice compared to control mice. FACS analysis of testicular germ cells revealed very high percentage of apoptotic cells (84\%) compared to control animals (2-3\%) indicative of reduced spermatogenic activity. In addition, a significant decrease in litter size (11.6\%-Control to 3.66\%-HFD) indicates that reproductive efficiency in high fat diet fed mice reduced to a great extent. The present study revealed high fat diet alters the fertility in male mice significantly and the first in this process appears to be apoptosis of testicular cells due to increase in ROS.
\end{abstract}

Key words: Apoptosis, High fat diet, Infertility, Obesity, Reproductive failure, Spermatogenesis.

\section{INTRODUCTION}

Although consuming balanced diet with right proportion of carbohydrates, fat and vitamins is desirable, it is not possible in the present day life situations. With the advent of fast foods, there has been an alarming increase in consumption of high fat diet mainly consisting of cheese and oily snacks. In the present day lifestyle of young adults whose diet is dominated by unsaturated and trans-fats, percentage of infertility in young couples is increasing gradually (Hussain et al. 2016; Jungheim et al. 2013). Several studies are directed towards investigating the role and significance of diet in alleviating the problem. In this regard, it is pertinent to note that the well established high fat diet consumption results in obesity with associated problems like diabetes, high cholesterol, cardio vascular diseases etc. and also results major biochemical changes which in turn leads to increase in ROS (Roza et al. 2016; Spencer et al. 2004). In the past decades, the adverse effects of obesity on female fertility have been well recognized. Some of the key effects are observed in terms of polycystic ovarian syndrome, menstrual disorder, miscarriage, lower conception rate and so on (Pasquali et al. 2003). Obesity is also known to have a role in male infertility by the way of decreased sperm count and sperm motility (Bakos et al. 2011, Hammoud et al. 2012). Several studies strongly attribute sperm DNA damage and intracellular reactive oxygen species, which in turn results in oxidative stress, with diet-induced obese male (Bakos et al. 2011. Fernandez et al. 2011). It is known that high body mass index and accumulation of excessive fat further increases oxidative stress along with the above mentioned factors.

Reactive oxygen species (ROS) are highly reactive oxidizing agents. Cells have a defense system under aerobic condition against ROS. There is a balance between antioxidants and pro oxidants in normal circumstances. When the body fails to eliminate excess ROS or an over production of ROS develops, it damages the tissues and cells (Tafuri et al. 2015). In addition, in terms of male reproductive system, consequential changes in semen factors such as the concentration, motility, viability and morphology of sperms have also been attributed to obesity (Martini et al. 2010. Shayeb et al. 2011). In this regard, studies on high-fat diet fed mice, apparent pathological changes in testicular tissue were indicated by seminiferous tubules that were small, atrophic and distorted and by damaged basement membrane (Bakos et al. 2011;Hammoud et al. 2012). However, even with the growing evidence of the role of obesity in impairing male fertility (Hammiche et al. 2012. Hammoud et al. 2008; Hofny et al. 2010), studies have failed to establish and document the relationship between the two (male infertility and obesity) distinctly(Jensen et al. 2004;Rybar et al. 2011). In support of this suggestion, clinical data from large-scale epidemiological studies was found to indicate a remarkable negative correlation between several semen factors, such as mentioned above, and body mass index (Chavarro et al.

*Corresponding author's e-mail: rosaj66@hotmail.com

${ }^{1}$ Department of Environmental Biotechnology, Bharathidasan University, Tiruchirappalli-620 024, Tamil Nadu, India.

${ }^{2}$ FACS Facility, Division of Biological Sciences, Indian Institute of Science, Bangalore-560 012, Karnataka, India

${ }^{3}$ Department of Animal Science, Bharathidasan University, Tiruchirappalli-620 024, Tamil Nadu, India 
2010; Fejes et al. 2005; Martini et al. 2010; Paasch et al. 2010; Shayeb et al. 2011). In other studies, data indicate that the alteration of sex hormone levels (such as increased testosterone and estradiol levels) in obese men is found to affect spermatogenesis (Chavarro et al. 2010,;Liu et al. 2014)

Over the years several studies have been carried out on the effects of high fat diet on metabolic changes and reproduction using rodent models (Graber et al. 1965; Harishankar et al. 2011), however, the sequences of changes which culminate in reproductive failure have been mostly conducted in relation to female reproductive system. In the present study an attempt has been made to document early changes which, ultimately result in failure of reproduction in male mice.

\section{MATERIALS AND METHODS}

Animals, housing and feeding: A total of 12 animals (4 weeks old male C57BL6 mice) for the study were obtained from the Central Animal Facility, Indian Institute of Science, Bangalore. All animals were weighed and divided into two groups of 6 each. Animals were housed in polycarbonate cage with corncobb (Sagar Industries, Bangalore) as bedding material. Animals were maintained under controlled environmental conditions with $12: 12 \mathrm{~h}$ light and dark cycle. The temperature was maintained at $22 \pm 2^{\circ} \mathrm{C}$ and humidity within a range of $30-70 \%$ throughout the study period. Bedding material along with cage was changed twice a week. The experiment was carried out for a period of 15 weeks.

Control animals were provided with standard pelleted rodent feed (Rayan Biotechnologies Pvt. Ltd., Hyderabad) and experimental animals with high fat diet (Research Diet HFD, D12492) for 15 weeks. All the animals were provided with purified filtered and UV irradiated water ad libitum during the entire period of the experiment. The experimental protocol was approved by the Institutional Animal Ethics Committee, Central Animal Facility, Indian Institute of Science and complied with guidelinesof Committee for the Purpose of Control and Supervision of Experiments on Animals (CPCSEA). Body weights of animals were recorded at the start of the experiment and every week subsequently till the completion of the study. Consumption of feed by animals which were provided 130 $\mathrm{g}$ of feed was calculated on weekly basis.

Estimation of serum glucose and cholesterol levels: Blood samples from animals were collected through retro-orbital plexus by using capillary tubes. The blood samples were allowed to coagulate at room temperature for one hour and subsequently centrifuged at $3500 \mathrm{rpm}$ for $15 \mathrm{~min}$ in a refrigerated centrifuge. The clear, non-haemolysed supernatant serum was aspirated using pipette and stored at $-20^{\circ} \mathrm{C}$ until further analysis. Serum glucose and cholesterol levels were analysed by Liquixx glucose and Liquixx cholesterol estimation kits (Erba Mannheim, Germany).
Estimation was carried out according to manufacturer's instructions.

Estimation of Reactive Oxygen Species (ROS) level in RBCs using DCFDA: Both high fat diet fed and controls animals were anesthetized and blood samples were collected through cardiac puncture for this study. Blood samples were immediately mixed with acid-citrate-dextrose (ACD) in the ratio of 5:1 (blood: $\mathrm{ACD}, \mathrm{v} / \mathrm{v})$. About $100 \mu \mathrm{l}$ aliquots of anti-coagulated blood were used to determine cell count in an automated hemo-analyser. Further, the remaining anticoagulated blood was then centrifuged at $3000 \mathrm{rpm}$ for 15 min to obtain RBCs. The cell count was determined in RBCs using a Neubauer chamber and adjusted to $5 \times 10^{8} \mathrm{cells} / \mathrm{mL}$ in the final suspension using Tyrode's buffer. Endogenous ROS generation in RBCs of control and high-fat diet fed mice blood was monitored using 2', 7' -dichlorofluorescin diacetate (DCFDA) staining. After isolation, cells were stained using DCFDA $(5 \mu \mathrm{M})$ for $30 \mathrm{~min}$, washed and analysed using FACS Calibur flow cytometer (BD Biosciences, USA). The signals were read at Ex $485 \mathrm{~nm}$, Em $535 \mathrm{~nm}$.

DNA analysis using FACS: At the end of the experiment, the mice were euthanizedand testes were collected. Single cell suspension of cells wasobtained for cell cycle analysis. Determination of number apoptotic cells was carried out using Propidium Iodide (PI) staining. Approximately 20,000 cells were acquired and was analysed using FACSDiva Version 6.1.3 program.

Tissue sampling and histological preparation: At the end of the study, animals were euthanized humanely and adipose tissue, fat tissue (Mann et al. 2014) and testes were collected and weighed immediately. The testes collected from control and HFD animals were fixed in Neutral Buffered Formalin (NBF) solution and processed for histological examination.

Mating studies: High fat diet fed male animals were allowed to mate with one control diet fed female for one week and the mating success was assessed by plug formation in the female. For estimating the mating efficiency, control male and female were also allowed to mate and compared with the studies carried with high fat fed animals. The success rate was assessed between these two.

Statistical analyses: All data were expressed as mean \pm SEM. Statistical analyses were done by using Graph Pad Prism (V5.0). Unpaired t-tests were done wherever required and values with $\mathrm{P}<0.05$ were considered significant.

\section{RESULTS AND DISCUSSION}

It was interesting to note that in spite of providing high fat diet for 15 weeks HFD, fed animals showed only a marginal increase $(0.12 \%)$ in body weight (Table 1$)$. In contrast, fat weight increased by 3 -fold and adipose tissue weight by1.4-fold. As expected HFD feeding led to 3.6-fold increase in blood glucose levels and cholesterol levels by 
Vol. Issue , ()

Table 1:

\begin{tabular}{lccc}
\hline Parameter & Normal Mice & High Fat Diet Mice & $P$ Value \\
\hline No. of Mice per group & 06 & 06 & \\
Initial weight (g) & $19.60 \pm 0.1581$ & $21.04 \pm 0.2599$ & 0.0036 \\
Final weight (g)(Week 15) & $22.50 \pm 0.1581$ & $25.12 \pm 0.1662$ & $<0.0001$ \\
Body weight gain (g) & $2.860 \pm 0.2337$ & $4.086 \pm 0.2907$ & 0.0118 \\
Serum glucose (mg/dL) & $76.33 \pm 4.609$ & $354.5 \pm 34.81$ & $<0.0001$ \\
Serum cholesterol (mg/dL) & $76.33 \pm 4.609$ & $120.4 \pm 5.767$ & 0.0002 \\
Serum Testosterone (ng/dL) & $2.167 \pm 0.1202$ & $65.40 \pm 4.993$ & 0.0002 \\
ROS generation (\%) & $19.68 \pm 1.235$ & $71.954 \pm 4.325$ & $<0.0001$ \\
Total Fat (g) & $0.3971 \pm 0.05776$ & $1.644 \pm 0.2101$ & $<0.0001$ \\
Adipose tissue (g) & $0.1543 \pm 0.01043$ & $0.3743 \pm 0.02852$ & $<0.0001$ \\
Percent & $2-3 \%$ & $84 \%$ & \\
apoptosis & $(52 \%$ diploid and & $(7.6 \%$ diploid and & \\
& $43 \%$ haploid cells) & $4.8 \%$ haploid cells) & $0.1686 \pm 0.01184$ \\
Testes weight $(\mathrm{g})$ & $0.1543 \pm 0.007825$ & $3.667 \pm 0.3333$ & $<0.3339$ \\
Percent Litter size & $11.67 \pm 0.3333$ & & $<0001$ \\
\hline
\end{tabular}

0.58 -fold. To check whether there was any excessive production of ROS in HFD fed mice, we analysed RBCs after separating from whole blood. ROS production showed $50 \%$ increase in HFD fed animals compared to controls. It can be seen from the Table 1 that in control diet fed mice nearly $52 \%$ diploid and $43 \%$ haploid cells and also between 2-3\% apoptosis were seen. However, in high fat diet fed animals $84 \%$ apoptotic cells were seen and only $7.6 \%$ diploid and $4.8 \%$ haploid cells were seen, which suggests that spermatogenesis is severely compromised. This in turn suggests that high fat diet ultimately induces cell death in testis. However, only a minimal change in testes weight was observed in HFD fed males compared to the controls. Following these changes, there was decrease in the seminiferous tubule diameter in the high fat fed mice. While nearly 4 tubules could be seen in one microscope (10X) field of the control diet mice only two tubules could be seen (Fig 1) in the high fat fed diet suggesting the decrease in tubule diameter, which is indicative of the decreased spermatogenesis. This could be due to decreased testosterone. However, this is not reflected in the actual serum testosterone levels in the high fat diet fed animals. As can be seen in the results observed (mean values: Control: $2.1 \mathrm{ng}$ / dL, HFD: $65.4 \mathrm{ng} / \mathrm{dL}$ ) in the high fat diet fed animals a very high and abnormal serum testosterone values were seen. The elevated increase in the testosterone levels may not be the effect of high fat diet alone. Differences in the metabolism of gut bacteria could explain some of the oddities observed in hormone metabolite levels as described in earlier studies (Dloniak and Holekamp, 2004). Further, difference in testosterone levels among individual animals should also be correlated with composition of gut bacteria (Goymann 2012). In addition, high testosterone levels observed could also be due to antibody cross-reactivity with hormone metabolites of different origins and/or metabolic association between testosterone and other metabolites (Gesquiere et al. 2014). Diet can alter the way hormones are metabolized, and different diets can change the amount of faecal bulk. Both phenomena can result in measurement artefacts that may seriously distort the estimation of hormone metabolite concentrations. In view of this, it is possible that the observed high values of testosterone may be due to artefacts in the high fat diet.

Mating characteristics of both high fat and normal diet fed male mice were observed (between 8-9 weeks). Animals from both groups were allowed to mate with normal diet fed female mice. The mating success (plug formation) was observed to be $75 \%$ less with high-fat diet fed males when compared to controls. Litter size in controls was found to be 10-12 whereas in HFD fed male it was only 2-3 (Table1). Survival rate of HFD fed male rats pups was only for one to two weeks.

The results of the present study, clearly indicate that one of the earliest changes in HFD induced obesity is increase in serum glucose and cholesterol. It is to be noted that although the increase in body weight is only marginal, there is a very significant $(\mathrm{P}<0.001)$ increase in adipose tissue weight within the time span of 15 weeks. It is known that increased adipose tissue results in an increase in reactive oxygen species, which has very damaging effect on membranes particularly sperm membrane. A consequence of this is increased apoptosis, which has been observed in
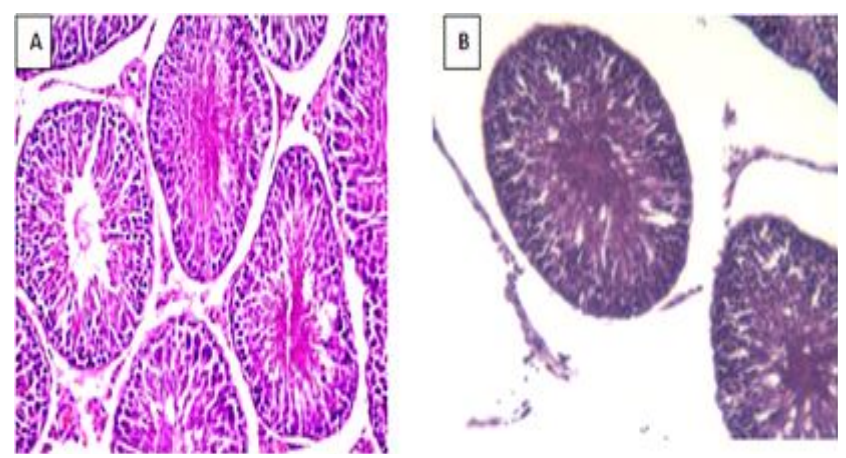

Fig 1: Histopathology of testis (A) mice fed with control diet (B) mice fed with HFD 
the present study, which resulted in a decrease in haploid cells and increased apoptosis as assessed by FACS analysis (Pozarowski 2004). Compared with the control group, the final body weight and weight gain were marginally higher in HFD mice. However, inspite of only marginal, decrease in testicular weight, the testis appeared small atrophic with distorted seminiferous tubules and destroyed basement membrane.

It is obvious from the results of present study that obesity has hazardous effects on reproductive system and fertility and exerts infertility via impaired mating and decline of successful pregnancy or low pregnancy rate combined with high mortality rate. A significant $(\mathrm{P}<0.001)$ decrease in litter size was reported in the HFD fed mice by Bakos et al. 2011.Studies have suggested that increase in intracellular reactive oxygen species and DNA damage of sperms is associated with diet-induced obese male. A high body mass index and fat accumulation promote the oxidative stress, which has been observed in the present study. Although, the reactive oxygen species are required for normal sperm function (Gesink Law et al. 2007), such as capacitation and acrosomal reaction, a negative impact on the sperm quality can be observed with increased levels of reactive oxygen species. Therefore, identifying and devising new strategies to counter the ill effects of obesity and high fat diet on male reproductive system is imperative. Some of the approaches to overcome the negative effects could be to restrict the use of high fat diet and consumption of antioxidants. Caloric restriction without compromising on nutrition is also a viable method to counter obesity (Mciver et al. 2012). These measures could help prevent complications arising out of obesity and help improve immune responses, at least in part, and antioxidant enzyme activities.

\section{CONCLUSION}

The results of the present study show that the high fat diet alters male fertility in mice. This study catalogues, step by step, the effect of high fat diet right from earliest, where the changes in terms of increased blood glucose and cholesterol levels observed, to the end point, where in it affects the sperm quality and male reproductive system as a whole. Increased adipose tissue and increase in reactive oxygen presence can be a cause leading to reproductive inefficiency.

\section{Conflict of Interest}

All authors declare no conflict of interest.

\section{ACKNOWLEDGEMENT}

Authors would like to thank Central Animal Facility (CAF), Indian Institute of Science (IISc), Bangalore; Dr Krishnaveni N, CAF, IISc, Bangalore.

\section{REFERENCES}

Bakos, H.W., Mitchell, M., Setchell, B.P. and Lane, M. (2011). The effect of paternal diet-induced obesity on sperm function and fertilization in a mouse model. Int J Androl. 34: 402-410.

Chavarro, J.E., Toth, T.L., Wright, D.L., Meeker, J.D. and Hauser, R. (2010). Body mass index in relation to semen quality, sperm DNA integrity, and serum reproductive hormone levels among men attending an infertility clinic. Fertil Steril. 93: 2222-2231.

Dloniak, S.M., French, J.A., Place, N.J., Weldele, M.L., Glickman, S.E. and Holekamp, K.E. (2004). Non-invasive monitoring of fecal androgens in spotted hyenas (Crocuta crocuta). General and Comparative Endocrinology. 135: 51-61.

Fejes, I., Koloszár, S., Szöllosi, J., Závaczki, Z. and Pál, A. (2005). Is semen quality affected by male body fat distribution? Andrologia. 37: 155-159.

Fernandez, C.D.B., Bellentani, F.F., Fernandes, G.S.A., Perobelli, J.E., Favareto, A.P.A., Nascimento, A.F., Cicogna, A.C. and Kempinas, W.D.G. (2011). Diet-induced obesity in rats leads to a decrease in sperm motility. Reprod Biol Endocrinol. 9: 32 .

Gesink Law, D.C., Maclehose, R.F. and Longnecker, M.P. (2007). Obesity and time to pregnancy. Hum Reprod. 22: 414-420.

Gesquiere, L.R., Ziegler, T.E., Chen, P.A., Epstein, K.A., Alberts, S.C. and Altmann, J. (2014). Measuring fecal testosterone in females and fecal estrogens in males: comparison of RIA and LC/MS/MS methods for wild baboons (Papio cynocephalus). Gen Comp Endocrinol. 204: 141-149.

Goymann, W. (2012). On the use of non-invasive hormone research in uncontrolled, natural environments: the problem with sex, diet, metabolic rate and the individual. Methods in Ecology and Evolution 3: 757-765.

Graber, C.D., O'neal, R.M. and Rabin, E.R. (1965). Effect of high fat diets on intestinal microflora and serum cholesterol in rats. $J$ Bacteriol. 89: 47-51.

Hammiche, F., Laven, J.S.E., Twigt, J.M., Boellaard, W.P.A., Steegers, E.A.P. and Steegers-Theunissen, R.P. (2012). Body mass index and central adiposity are associated with sperm quality in men of subfertile couples. Hum Reprod. 27: $2365-2372$.

Hammoud, A.O., Carrell, D.T., Gibson, M., Peterson, C.M. and Meikle, A.W. (2012). Updates on the relation of weight excess and reproductive function in men: sleep apnea as a new area of interest. Asian J Androl. 14: 77-81.

Hammoud, A.O., Wilde, N., Gibson, M., Parks, A., Carrell, D.T. and Meikle, A.W. (2008). Male obesity and alteration in sperm parameters. Fertil Steril. 90: 2222-2225.

Harishankar, N., Kumar, P.U., Sesikeran, B. and Giridharan, N. (2011). Obesity associated pathophysiological \& histological changes in WNIN obese mutant rats. Indian J Med Res. 134: 330-340.

Hofny, E.R.M., Ali, M.E., Abdel-Hafez, H.Z., Kamal, E.E.-D., Mohamed, E.E., Abd El-Azeem, H.G. and Mostafa, T. (2010). Semen parameters and hormonal profile in obese fertile and infertile males. Fertil Steril. 94: 581-584.

Hussain, M.A., Abogresha, N.M., Hassan, R., Tamany, D.A. and Lotfy, M. (2016). Effect of feeding a high-fat diet independently of caloric intake on reproductive function in diet-induced obese female rats. Arch Med Sci. 12: 906-914. 


$$
\text { Vol. Issue , () }
$$

Jensen, T.K., Andersson, A.-M., Jørgensen, N., Andersen, A.-G., Carlsen, E., Petersen, J.H. and Skakkebaek, N.E. (2004). Body mass index in relation to semen quality and reproductive hormones among 1,558 Danish men. Fertil Steril. 82: 863-870.

Jungheim, E.S., Travieso, J.L. and Hopeman, M.M. (2013). Weighing the impact of obesity on female reproductive function and fertility. Nutr Rev 71: S3-8.

Liu, Y., Zhao, W., Gu, G., Lu, L., Feng, J., Guo, Q. and Ding, Z. (2014). Palmitoyl-protein thioesterase 1 (PPT1): an obesity-induced rat testicular marker of reduced fertility. Mol Reprod Dev 81: 55-65.

Mann, A., Thompson, A., Robbins, N. and Blomkalns, A.L. (2014). Localization, identification, and excision of murine adipose depots. $J$ Vis Exp 94. Doi: 10.3791/52174.

Martini, A.C., Tissera, A., Estofán, D., Molina, R.I., Mangeaud, A., De Cuneo, M.F. and Ruiz, R.D. (2010). Overweight and seminal quality: a study of 794 patients. Fertil Steril. 94: 1739-1743.

Mciver, C.M., Wycherley, T.P. and Clifton, P.M. (2012). MTOR signaling and ubiquitin-proteosome gene expression in the preservation of fat free mass following high protein, calorie restricted weight loss. Nutr Metab (Lond) 9: 83.

Paasch, U., Grunewald, S., Kratzsch, J. and Glander, H.-J. (2010). Obesity and age affect male fertility potential. Fertil Steril. 94: 2898-2901.

Pasquali, R., Pelusi, C., Genghini, S., Cacciari, M. and Gambineri, A. (2003). Obesity and reproductive disorders in women. Hum Reprod Update 9: 359-372.

Pozarowski P, D.Z. (2004). Analysis of cell cycle by flow cytometry. Methods Mol Biol. 281: 301-311.

Roza, N.A.V., Possignolo, L.F., Palanch, A.C. and Gontijo, J.A.R. (2016). Effect of long-term high-fat diet intake on peripheral insulin sensibility, blood pressure, and renal function in female rats. Food Nutr Res 60: 28536.

Rybar, R., Kopecka, V., Prinosilova, P., Markova, P. and Rubes, J. (2011). Male obesity and age in relationship to semen parameters and sperm chromatin integrity. Andrologia. 43: 286-291.

Shayeb, A.G., Harrild, K., Mathers, E. and Bhattacharya, S. (2011). An exploration of the association between male body mass index and semen quality. Reprod Biomed Online 23: 717-723.

Spencer, M.W., Mühlfeld, A.S., Segerer, S., Hudkins, K.L., Kirk, E., Leboeuf, R.C. and Alpers, C.E. (2004). Hyperglycemia and hyperlipidemia act synergistically to induce renal disease in LDL receptor-deficient BALB mice. Am J Nephrol. 24: 20-31.

Tafuri, S., F.C., Eugenio Luigi Iorio, Luigi Esposito and Natascia Cocchia. (2015). Reactive Oxygen Species (ROS) and Male Fertility. In New Discoveries in Embryology, D.B. Wu, ed., InTech. Doi: 10.5772/60632. 